\title{
RTChoke: Efficient Real-Time Traffic Chokepoint Detection and Monitoring
}

\author{
Vikram Munishwar \\ State University of New York, Binghamton. \\ vmunish1@cs.binghamton.edu
}

\author{
Vinay Kolar, Praveen Jayachandran, Ravi Kokku \\ IBM Research, India \\ \{vinkolar,praveen.j,ravkokku\}@in.ibm.com
}

\begin{abstract}
We present a novel efficient adaptive sensing and monitoring solution for a system of mobile sensing devices that support traffic monitoring applications. We make a key observation that much of the variance in commute times arises at a few congestion hotspots, and a reliable estimate of congestion can be obtained by selectively monitoring congestion just at these hotspots. We design a smartphone application and a backend system that automatically identifies and monitors congestion hotspots. The solution has low resource footprint in terms of both battery usage on the sensing devices and the network bytes used for uploading data. When a user is not inside any hotspot zone, adaptive sampling conserves battery power and reduces network usage, while ensuring that any new hotspots can be effectively identified. Our results show that our application consumes $\mathbf{4 0 - 8 0 \%}$ less energy than a periodic sampling system for different routes in our experiments, with similar accuracy of congestion information. The system can be used for a variety of applications such as automatic congestion alerts to users approaching hotspots, reliable end-to-end commute time estimates and effective alternate route suggestions.
\end{abstract}

\section{INTRODUCTION}

Traffic congestion is a problem that most urban locales struggle to grapple with. In India, vehicle population has grown by over a 100 times in the last 50 years, while length of roads has increased by only 8 times [1] during this period. It is estimated that 4.8 billion hours of time and 1.9 billion gallons of fuel were wasted due to congestion in 2011 in the US alone [2]. While governments strive to curtail congestion through various methods such as car-pooling incentives and congestion pricing, the onus has fallen on individual users to best cope with it.

Recent research has focused on participatory sensing techniques for congestion detection as an alternative to infrastructure based systems that are often expensive and hard to maintain [3], [4], [5], [6]. In participatory sensing, users willingly contribute information from sensors they already own (such as those in their cell phones), which can be aggregated and analyzed at a central server. Here, mobile phones essentially behave as mobile sensors uploading information from where they are at any point in time. Unfortunately, proposed techniques using participatory sensing decouple collection of data from the use of the data, and often use periodic sampling and uploading from mobile phones. Depending on the period of sampling, this often results in oversampling in dense regions and times than required, leading to increased battery and

978-1-4244-8953-4/11/\$26.00 (C) 2015 IEEE network bytes usage on user's mobile devices.

To this end, we formulate the following problem at an abstract level: given a set of mobile sensor devices, how do we collect GPS location information from them with low resource footprint, while deriving similar insights as uniform fine-grained sampling? We build on the observation that it is sufficient to selectively monitor certain key congestion hotspots. Our approach is to "couple" data collection with the use or utility of the data collected, i.e., more data is collected in places and at times where there is more utility than at other places and times. In fact, we observe through measurement studies that most of the speed variation with time on a person's road trajectory is contributed by certain key hotspots. Key challenges, however, are to define what a congestion hotspot is in a generic sense, and to identify a hotspot reliably. Furthermore, congestion is a dynamic phenomenon, i.e., congestion hotspots may appear and disappear with time (e.g., due to accidents, construction, week-day office hours).

We design and implement a system RTChoke that consists of an Android application and a back-end analyzer that can automatically detect and monitor congestion hotspots. An adaptive sampling approach ensures that the emergence of new hotspots can be detected quickly as soon as they "become" hotspots, while also conserving critical battery power when the user is not driving within a hotspot zone. Further, a lightweight decision tree that uses a mix of sensors is used to detect if a user is driving, to automatically trigger location tracking using GPS and data upload to a central server. The central server utilizes the data uploaded by different smartphones and detects hotspots. We demonstrate that the system can make accurate estimates of user commute times and suggest efficient alternate routes and commute start times, based only on information gathered at the hotspots, consuming only half the battery power compared to uniform monitoring tools such as Google Maps [7]. Our results are consistent across users of our Android application in a large city in a developing nation, as well as in experiments using taxicab traces for San Francisco [8].

In summary, this paper makes two key contributions:

- Building on the observation that it is sufficient to monitor certain key congestion hotspots, we first explore how to define a hotspot in a generic sense, and then describe how to continuously and automatically monitor and detect hotspots. 
- We design an adaptive monitoring technique for mobile phones that detects if the user is driving a vehicle, and monitors GPS location or speed more frequently as we approach closer to the currently active hotspots, and less frequently when away from the hotspots; the farther we are from the hotspot, the lower is the sampling frequency. This effectively couples the data collection to their use, ensuring energy efficiency.

The rest of the paper is organized as follows. Section II formulates the problem, introduces the notion of hotspots, and discusses related work. Section III describes the design and implementation of RTChoke. Section IV provides analysis of our proposed approach. Section $\mathrm{V}$ presents performance evaluation of RTChoke. Section VI concludes the paper.

\section{PROBLEM Formulation}

In this section, we first motivate our solution approach through a simple experiment that shows the presence of traffic congestion hotspots. We then provide a formal definition of a hotspot, and discuss related work.

\section{A. Motivation}

Twelve employees from our organization used the smart phone application developed by us for two months, contributing location and speed information from their trips to and from office - hereafter we refer to this office location as TechPark (marked with a rectangle in the Fig. 1). The figure shows a heat map of average speed (points indicate only speeds less than $30 \mathrm{kmph}$ ) on a road stretch just outside the TechPark across all these trips. Fig. 1 shows that the average speed just outside the TechPark is mostly $5-15 \mathrm{kmph}$ during the commute hours, indicating the presence of a chokepoint or a hotspot. The speed on the same road segment is greater than $30 \mathrm{kmph}$ during afternoons and late nights.

To illustrate the nature and effect of congestion on commute times, we consider the data from a single user (other users demonstrate similar trends). Over a period of two months, the user arrived at office each day at some time between 8:00 AM and 11:30 AM along the trajectory shown in Fig. 2 We studied the commute time to traverse the $2 \mathrm{~km}$ of his commute closest to the TechPark (referred to as Hotspot Zone in Fig. 2), and compared this with the time taken to traverse the rest of the distance from his home, which was about $6 \mathrm{~km}$. Fig. 3 shows these two time-splits, namely, within the hotspot zone and outside the hotspot zone, for different times at which the user entered the hotspot zone. Outside the hotspot zone, the user covered a distance of $6 \mathrm{~km}$ in about 8 minutes on average. In contrast, the last $2 \mathrm{~km}$ of his commute took more than 15 minutes on average. Another interesting observation is that the variance in commute times is very low for the trip up to the hotspot, while the variance is considerably higher inside the hotspot zone.

The above observations form the primary motivation for our work. We ask ourselves the following questions. How do we define and automatically detect such congestion hotspots? Can we develop a smart phone application that will require no active user participation, consume minimal battery power, and yet provide automatic and accurate congestion estimates? Is it possible to tune the system parameters to meet user's power budget constraints? We built a system that answers these questions in the affirmative.

We define a congestion hotspot as a geo-spatial region (specifically, a road segment of a certain length) with the following properties:

- Current average speed $\leq \tau \times$ reference speed, where $\tau$ is a predefined congestion threshold, and reference speed is a reasonable achievable speed in the region. In our implementation, we use the maximum speed observed as the reference speed.

- The region shows high temporal variance in average speed.

- The congestion at all points within the hotspot region is similar at all times.

While several studies exist that define other notions of congestion zones [9], [10], we find that our simple definition is sufficient in practice as typical congestion situations are contained within this zone.

B. Related Work

GPS and other sensors are widely used to achieve low-cost traffic monitoring. In this section, we briefly discuss recent studies, and describe how we advance the state-of-the-art.

Participatory Sensing: Participatory traffic monitoring can be broadly classified into vehicle-mounted and smart-phone based sensing. In vehicle-mounted sensing, location data is collected from moving objects that are mounted with GPS and other sensors [11], [12]. Examples of such sensing include: (a) Green GPS that computes fuel-efficient routes [13], (b) Pothole Patrol accesses road surface conditions [14], and (c) Taxi durations and fare estimation [15].

In smart-phone based sensing, users are generally provided with an application that automatically collects data from the smart-phone sensors [16]. Mobile Millenium [3] and Nericell [6] utilize multiple sensors on smart-phone, such as GPS, cellular connection and accelerometers, that detect traffic delays and congestion. A transit tracking system enables periodic GPS sampling if the user is moving in a vehicle, and then detects congestion is developed in [4], [5].

Similar to the above studies, RTChoke is a participatory sensing approach with minimal resource footprint, and minimal user intervention, which requires the user to primarily install and start the application once. Other applications providing similar services include Google Maps [7] and Waze [17], but both of them do not adapt based on the data utility. Moreover, they require significant user participation.

Traditional Road Traffic Engineering Models: There is a large body of work in traffic control systems for detecting congestion, such as three-phase theory [9], Forecasting of Traffic Objects (FOTO) and Automatic Tracking of Moving Traffic Jams (ASDA) [10]. These systems typically require large amount of historical data to build reliable models, do not work for dynamic unpredictable hotspots, and require that traffic does not evolve significantly from the data for the models to remain valid. 


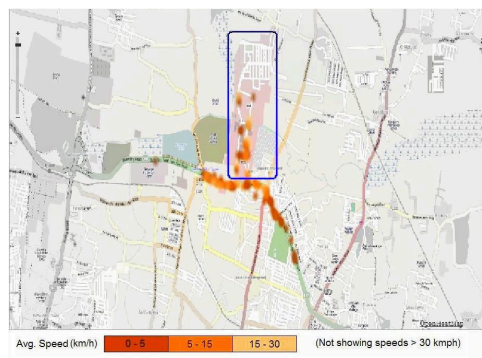

Fig. 1. Heat map of average speeds from commute routes of employees

\section{DESIGN AND IMPLEMENTATION}

Given a road network, and a system of mobile devices traveling on the road network, the goal of RTChoke is to (1) identify traffic congestion hotspots dynamically using data uploaded from the mobile devices, and (2) monitor the congestion hotspots continuously while being efficient in terms of battery and network resources utilized on the mobile devices. Several applications such as automatic congestion alerts to travelers, end-to-end commute time predictions, alternate route suggestions can be instantiated over RTChoke.

For tractability, RTChoke considers that a road network is broken down into road segments (including lines and curves) [18]. Each road segment is a geospatial region that is tagged as a hotspot when it satisfies the conditions described in Section III To achieve the conflicting goals of maintaining reliability of tagging a region as a hotspot, and reducing the overhead of maintaining too many road segments, one needs to strike a tradeoff on the length of the road segments. For instance, longer segments (especially on highways) reduce the total number of road segments, but may be inaccurate in capturing congestion if only parts of the segments are congested during busy hours. To balance this tradeoff, RTChoke assumes that these segments are of $10 \mathrm{~s}$ to $100 \mathrm{~s}$ of meters. One sideeffect of this tradeoff, however, is that adjacent road segments can be completely correlated at all times; in such scenarios, RTChoke considers only one of the correlated segments as a representative hotspot, and avoids maintaining information or monitoring the other segments.

\section{A. Solution Overview}

We now describe the overall architecture and process of RTChoke, pictorially shown in Figure 4. A smart-phone runs an application in the background to selectively sample GPS and upload the location data to the server in real time. On the phone, an activity classifier is invoked every 30 seconds to detect a user's activity. If the user is driving, it invokes Congestion Hotspot Retriever to obtain current congestion hotspot information from server, and Sampling Rate Estimator to adapt GPS sampling interval based on the nearest congestion hotspot. Once a location sample is obtained from GPS, it is uploaded to the server by the Data Uploader. The server receives location updates and passes them to a Daemon. The Daemon validates each location and maps it to a point on the road as indicated by the Map-Matching

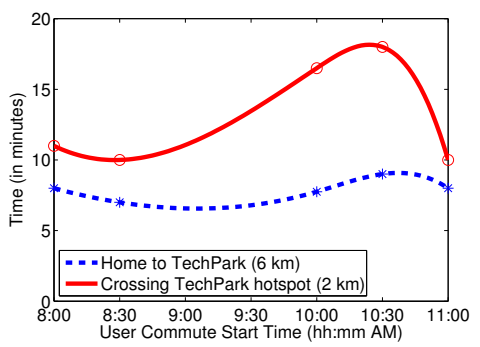

Fig. 3. Split of time taken outside hotspot zone and within hotspot zone

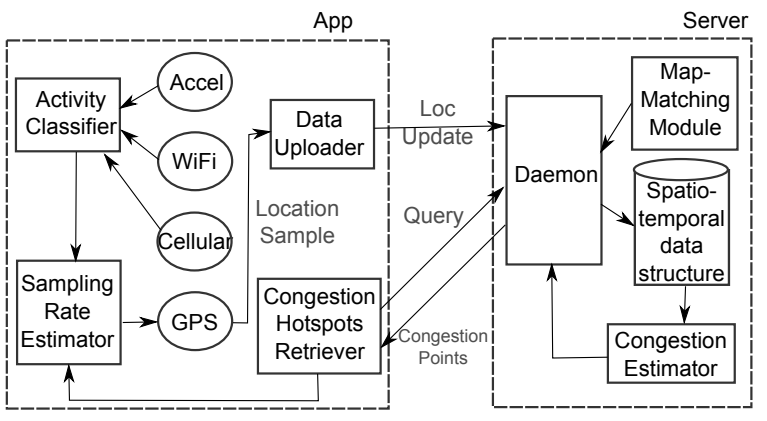

Fig. 4. Overall system architecture.

Module. Map-matching corrects the GPS sampling errors and snaps the location samples to the road-paths traveled. In the current implementation, we use a Hidden Markov based mapmatching [19]. User's information along with the updated location is placed into a spatio-temporal data structure, which is used by the Congestion Estimator to detect congestion hotspots.

RTChoke's functionality can be mainly categorized into two components: (1) detecting that a road segment is a hotspot, and (2) monitoring at the hotspot frequently, while also monitoring non-hotspots less frequently to be able to detect new hotspots. We describe each components now.

\section{B. Hotspot Detection}

Hotspots can be detected using different approaches. A majority of them rely on parameters such as maximum speed limit on a road or number of lanes [10]. While such parameters are easily available for roads in developed countries, they are either not defined, or changing over time, or not followed strictly in the developing countries. Further, these parameters are not sufficient to detect congestion caused by occasional incidents such as accidents or minor road works. Thus, to detect new congestion hotspots automatically, we do not assume knowledge of any such parameters. Instead, we use dynamic speed information received at the server for each road-segment over a long term to infer a reference speed, and view the current speed samples in relation to the reference speed.

RTChoke also recognizes that congestion is a dynamic phenomenon on a road segment, which varies based on the the spatial location of the road segment, time-of-the-day and 
unpredictable road incidents such as accidents and construction. We now describe how RTChoke accounts for the spatiotemporal features of congestion and road dynamics to tag the road as a hotspot.

1. Accounting for temporal variability of speeds: The speeds on the roads vary based on temporal aspects such as time-of-the-day. To capture these temporal effects we divide a day into 48 equal time-bins, each representing 30 minutes. For each road segment, we capture the number of samples received and average speeds. The server also maintains a maximum speed per segment across all times of the day. At each bin, we categorize congestion hotspots using low-, medium-, and high-congestion levels, similar to [9].

2. Hotspot Marking: A time-bin of a road-segment can exist in three states: uncongested, possible hotspot and hotspot. We initially set all road-segment bins to uncongested state. As the user data trickles down to the server, the server updates the average speeds. Based on the aggregated values, the server classifies the road as a into one of the three states.

Road segments may also experience flash-congestion due to incidents such as accidents or temporary obstructions. Automatic detection of flash hotspots is valuable since it can provide early warnings to users approaching a possible bottleneck or traffic regulators. To detect flash congestion, it is important to have a bounded sampling interval that is not larger than the time the user may take to cross the incident zone. When multiple recent samples with low speeds are obtained, a server tags the road-segment as possible hotspot. The server requests clients to increasingly sample road segments to conclude if the possible hotspot segment is a hotspot. We analyze the time required for flash-congestion detection in Section IV-B

3. Separating hotspots from inherently low-speed roads: Different roads may have different speed signatures depending on the road size and road-surface conditions such as speedbumps and pot-holes. Thus, low average speeds do not necessarily imply that the road-segment is a congestion hotspot. We distinguish congestion hotspots from roads with inherently low speeds.

For each road-segment, we keep track of the maximum speed, $V_{\max }$ observed in the past. We then tag a segment as hotspot if the current average speed for the time-bin is lower than a threshold $T_{\text {hot }}=0.25 \frac{V_{\max }}{2}$. We set two more thresholds: $T_{\text {med }}=0.5 \frac{V_{\max }}{2}$ and $T_{\text {low }}=0.75 \frac{V_{\max }}{2}$. We tag the segment as medium- or low-congestion if the speeds are between $\left(T_{\text {hot }}, T_{\text {med }}\right]$ or $\left(T_{\text {med }}, T_{\text {low }}\right]$, respectively. We obtain these thresholds based on observations made from experiments at TechPark.

\section{Hotspot Monitoring}

For energy efficiency and minimizing the network usage, the application running on a smart phone detects when the user is mobile, and only then starts the monitoring activity, and it adapts the monitoring frequency based on the distance from the hotspot. If multiple hotspots are close to the mobile device, the closest hotspot determines the monitoring frequency.

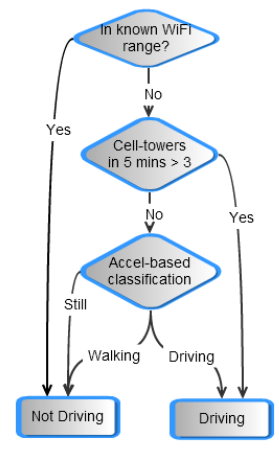

(a)

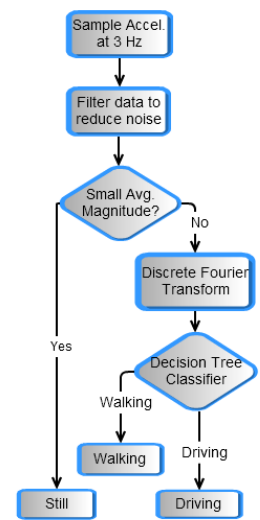

(b)
Fig. 5. Activity classification: driving vs. not driving. (a) Activity classification using WiFi, GSM, and accelerometer sensors; (b) Accelerometer-based classification.

1) Decision-Tree based Activity Detection: Activity detection allows the system to be completely automated, thus not requiring active user participation; this is an important design goal for RTChoke to ensure that people continue to use the application for a long time. Activity detection should accurately identify if a user is driving. False-positives in detection leads to excessive energy drain since the app will sample when the user is not driving (which is generally most of the day). False-negatives lead to loss of valuable data at the server side to detect hotspots.

We use an energy-aware combination of approaches for activity detection [4], [20], and switch-on and sample GPS only when the user is most probably driving. Figure 5(a) shows our classification mechanism. We employ a decision tree where we first attempt to find clues of vehicular movement without sensing. Specifically, we first check if the user is within the range of any known WiFi (office/home) access points. In such a case, the user is most likely not in a vehicle. Otherwise, we check the number of GSM cell-towers associated with in a given time window; if the number of towers is more than three in a short amount of time, we detect that the user is moving. Otherwise, we use accelerometer readings to determine if the user is driving (as shown in Figure [5(b)] [4].

2) Utility-aware Sampling: Once the activity classifier detects that the user is moving in a vehicle, GPS sampling is enabled. However, the utility of GPS samples vary spatiotemporally, based on the current distribution of hotspots. Each phone periodically polls the server when the user is driving, to obtain a current list of congestion hotspots, where each congestion hotspot is defined in terms of its latitude and longitude based location, and approximate distance for congestion spread, $d_{\mathrm{in}}$. Based on this information, the phone selects the next GPS sampling interval $y$ as: 


$$
\begin{aligned}
y & =S_{\min }, \quad \text { if } d \leq d_{\mathrm{in}} \\
& =S_{\min }+\left(1-\frac{d_{\mathrm{in}}}{d}\right) *\left(S_{\max }-S_{\min }\right), \text { if } d>d_{\mathrm{in}}
\end{aligned}
$$

where $d$ is the distance between the phone user and the closer of the end points of the nearest hotspot, and $S_{\min }$ and $S_{\max }$ are the minimum and maximum sampling interval for GPS. If the user is within the congestion zone, then GPS is sampled at higher frequency. Otherwise, GPS is sampled adaptively, as the data becomes less useful. Values of $S_{\min }$ and $S_{\max }$ can be selected based on a power-budget for the application, which is described in more detail in Section IV. $S_{\text {max }}$ provides a bound on the GPS sampling interval, which is useful in detecting new congestion hotspots.

The system supports querying about the congestion level at a given location and at a given time. The location data uploaded by the phone is recorded in a spatio-temporal data structure. We use road network information to maintain spatial information [18]. To maintain temporal congestion information, we record the speed on roads at different times of the day. This allows the system to notify details such as hotspot location during a particular time of the day, and time required to cross it to the user.

We now describe the design goals of the system, certain key features, followed by an overview of the various system components.

\section{Design Goals}

1) Require no active human participation: Existing traffic monitoring tools, such as Google maps [7] or Waze [17], require active user participation to start or stop the application, query for directions, or to spatiotemporally annotate events. While such systems are accurate, they are severely constrained as users are not always proactive. A fully automated system enables large and ubiquitous user is likely to attract more users. However, such a automated system places various design challenges to accurately detect if the user is driving a vehicle and subsequently enable a location tracking service.

2) Energy efficiency without compromising accuracy: Congestion detection requires GPS sampling and network transmission, which are energy intensive. RTChoke should have minimal energy consumption so that the user is not required to frequently charge the smart-phone battery.

3) Minimize network cost: The sampled location data needs to be uploaded to a server in real-time, only if deemed pertinent. If the data exchange is high, users do not participate willingly in the data collection process - especially when the mobile users pay per byte for cellular data connection.

4) Limited processing on the phones: Phones are required to detect if the user is driving or not, and accordingly start/stop GPS sampling and uploading. However, phones have limited processing capacity. Thus, lightweight processing techniques is preferred with minimal loss of accuracy. Similarly, scalar-sensors are be preferred over audio/video sensors that require complex data processing operations on the phones.

5) Ability to answer spatio-temporal queries: The system should be able to aggregate data to detect and monitor congestion hotspots, and respond to spatio-temporal queries such as optimal time for commute, providing congestion estimates for a particular location and/or time of day, etc.

\section{E. Design Features}

We next highlight key features of our design

1) Utility-Aware Data Sampling: Traditional sensor networks such as intrusion detection, and also existing traffic monitoring systems [7], [17] are "sample, then analyze"systems. Sensors uniformly collect the samples and later analyze the collected data. Such a system has the drawback of not analyzing the possible utility of the data before sampling and transmitting. We adopt an efficient "analyze and sample simultaneously" strategy where we analyze the potential utility of data before sampling, thus coupling the data collection with the possible utility of the data. In our system, the utility of data is ascertained using a few low-cost sensors combined with knowledge of proximity of the user to current congestion hotspots.

2) Energy-Efficient Adaptivity: Automatic congestion detection requires expensive GPS sampling. RTChoke saves energy by using a combination of low-energy sensing using $\mathrm{WiFi}, \mathrm{GSM}$ and accelerometer sensors. In addition, we automatically assess the utility of each data sample based on how much the sample can contribute towards accurately monitoring or detecting a hotspot. Our utility function considers various aspects such as distance from the nearest hotspot and last sampled time.

3) Automatic Congestion hotspot Detection and Monitoring: Sampled location data from the smart-phone app is uploaded to the server in real-time. Not all of the GPS data is accurate - the location estimate could be erroneous or stale. Therefore, we first cleanse the GPS data [20], and accurate estimate the user location using techniques such as mapmatching [19].

The system is also capable of detecting flash congestion, such as those created due to an accidents or construction. Furthermore, the system distinguishes between congested roads and roads where vehicles cannot travel at high speeds due to, say, speed bumps and potholes.

4) Spatio-Temporal Awareness: The system supports querying about the congestion level at a given location and at a given time. The location data uploaded by the phone is recorded in a spatio-temporal data structure. We use road network information to maintain spatial information [18]. To maintain temporal congestion information, we record the speed on roads at different times of the day. This allows the system 
to notify details such as hotspot location during a particular time of the day, and time required to cross it to the user.

\section{ANALYSis}

In this section, we analyze the utility-aware adaptive sampling and new hotspot detection approaches.

\section{A. Energy budget for Adaptive Sampling}

We model the energy consumption of adaptive sampling, and then use the analysis to choose appropriate values for the parameters in our adaptive sampling approach, that will ensure that the application does not consume more energy than stipulated.

\begin{tabular}{|c|c|}
\hline Symbol & Description \\
\hline$d_{\text {in }}$ & Distance inside congestion zone \\
$d_{\text {out }}$ & Distance outside congestion zone \\
$v_{\text {in }}$ & Avg. speed inside congestion zone \\
$v_{\text {out }}$ & Avg. speed outside congestion zone \\
$S_{\min }$ & Min. sampling interval \\
$S_{\max }$ & Max. sampling interval \\
$e_{\text {in }}$ & Energy per sample inside congestion zone \\
$e_{\text {out }}$ & Energy per sample outside congestion zone \\
\hline
\end{tabular}

TABLE I

NOTATION USED IN ANALYSIS. $y_{i}$ denote the sampling interval after the $i^{\text {th }}$ sample. It is safe to assume that the first sample outside the congestion zone is taken right at the edge of the zone, so $x_{1}=0$. Therefore, $y_{1}=S_{\min }$. We compute the distance covered and sampling interval for the subsequent samples as follows.

$$
\begin{array}{r}
x_{2}=v_{\text {out }} S_{\text {min }} ; \quad y_{2}=\frac{S_{\max } x_{2}+d_{\text {in }} S_{\text {min }}}{d_{\text {in }}+x_{2}} \\
=S_{\min }\left(\frac{d_{\text {in }}+v_{\text {out }} S_{\max }}{d_{\text {in }}+v_{\text {out }} S_{\min }}\right) \\
x_{3}=x_{2}+v_{\text {out }} y_{2}=v_{\text {out }} S_{\min }\left(1+\frac{d_{\text {in }}+v_{\text {out }} S_{\text {max }}}{d_{\text {in }}+v_{\text {out }} S_{\text {min }}}\right)
\end{array}
$$

Substituting for $x_{3}$ in Equation 2 and simplifying,

$$
\begin{aligned}
y_{3} & =S_{\min } \frac{\left(d_{\text {in }}+v_{\text {out }} S_{\text {max }}\right)^{2}+d_{\text {in }} v_{\text {out }} S_{\min }+v_{\text {out }}^{2} S_{\min } S_{\text {max }}}{\left(d_{\text {in }}+v_{\text {out }} S_{\text {min }}\right)^{2}+d_{\text {in }} v_{\text {out }} S_{\min }+v_{\text {out }}^{2} S_{\min } S_{\max }} \\
& >S_{\min } \frac{\left(d_{\text {in }}+v_{\text {out }} S_{\text {max }}\right)^{2}}{\left(d_{\text {in }}+v_{\text {out }} S_{\text {min }}\right)^{2}}
\end{aligned}
$$

as $\frac{a_{1}+b}{a_{2}+b}>\frac{a_{1}}{a_{2}}$, for $a_{1}, a_{2}, b>0$. Using these as basis step, it can be shown using induction on the number of samples $n_{\text {out }}$ that,
The notation used in the analysis is presented in Table $\mathbb{I}$ We make the following assumptions for our analysis. While these make the analysis simplistic, it helps us make intelligent choices for the values of parameters in our adaptive sampling approach. We consider a user who starts from the congestion hotspot and travels along a straight path to their destination covering a distance $d_{\text {in }}$ within the congestion zone first and then a distance $d_{\text {out }}$ outside the congestion zone (the analysis is equally applicable for users traveling towards the congestion hotspot). We assume that the average speed within the congestion zone is $v_{\text {in }}$ and that the user moves with a uniform speed of $v_{\text {out }}$ outside the congestion zone. Within the congestion zone, the application samples GPS values with a constant sampling interval of $S_{\min }$. Outside the congestion zone, we use the sampling interval as shown in Equation 1 Let $x=d-d_{\text {in }}$ denote the distance covered by the user outside the congestion zone at the instant a sample was taken. Simplifying Equation 1. we get the sampling interval $y$ :

$$
y=\frac{S_{\max } x+d_{\text {in }} S_{\min }}{d_{\text {in }}+x}
$$

We now bound the total energy used by the application during the course of the user's trip. The number of samples taken within the congestion zone is,

$$
n_{\text {in }}=\frac{d_{\text {in }}}{v_{\text {in }}} \frac{1}{S_{\text {min }}}
$$

We next determine an upper bound on the number of samples outside the congestion zone. Let $x_{i}$ denote the distance covered up to the $i^{\text {th }}$ sample outside the congestion zone and

$$
\begin{aligned}
x_{n_{\text {out }}} \geq & v_{\text {out }} S_{\min }\left[1+\left(\frac{d_{\text {in }}+v_{\text {out }} S_{\text {max }}}{d_{\text {in }}+v_{\text {out }} S_{\text {min }}}\right)+\left(\frac{d_{\text {in }}+v_{\text {out }} S_{\text {max }}}{d_{\text {in }}+v_{\text {out }} S_{\min }}\right)^{2}\right. \\
& \left.+\ldots+\left(\frac{d_{\text {in }}+v_{\text {out }} S_{\text {max }}}{d_{\text {in }}+v_{\text {out }} S_{\min }}\right)^{n_{\text {out }}-2}\right] \\
= & v_{\text {out }} S_{\min } \frac{\left(\frac{d_{\text {in }}+v_{\text {out }} S_{\max }}{d_{\text {in }}+v_{\text {out }} S_{\min }}\right)^{n_{\text {out }}-1}-1}{\frac{d_{\text {in }}+v_{\text {out }} S_{\max }}{d_{\text {in }}+v_{\text {out }} S_{\min }}-1}
\end{aligned}
$$

In order to bound the number of samples needed to cover a distance $d_{\text {out }}$ outside the congestion zone, we require that $x_{n_{\text {out }}-1}<d_{\text {out }} \leq x_{n_{\text {out }}}$. Substituting $r=\frac{d_{\text {in }}+v_{\text {out }} S_{\max }}{d_{\text {in }}+v_{\text {out }} S_{\min }}$ and solving for $n_{\text {out }}$,

$$
n_{\text {out }}=1+\left\lceil\log _{r}\left(1+\frac{d_{\text {out }}(r-1)}{v_{\text {out }} S_{\text {min }}}\right)\right\rceil
$$

Although, there are other computations that execute periodically, such as evaluating the decision tree to determine if the user is driving, for simplicity we assume that $e_{\mathrm{in}}$ and $e_{\text {out }}$, the average energy values consumed per sample inside and outside the congestion region, respectively, is uniform across all samples (this includes energy used for sensing, computation, and communication). Hence, the total energy used by the application for the entire trip can be determined as,

$$
E=n_{\text {in }} e_{\text {in }}+n_{\text {out }} e_{\text {out }}
$$

Based on average values from our local city experiments, we obtained the average speed within the congestion zone as $v_{\text {in }}=2 \mathrm{~m} / \mathrm{s}(16.67 \mathrm{kmph})$, and the average speed outside the congestion zone as $v_{\text {out }}=12.5 \mathrm{~m} / \mathrm{s}(45 \mathrm{kmph})$. The energy 
consumed per sample were obtained as, $e_{\mathrm{in}}=0.4 J$ and $e_{\text {out }}=7 \mathrm{~J}$ (note that $e_{\text {out }}$ is for a much larger time period as samples are obtained less frequently). We plot the energy as a function of the maximum sampling interval (as derived in Equation 5, for different values of the congestion zone radius $d_{\text {in }}$ and different minimum sampling intervals in Figure 6 , assuming that the total distance covered by the user in the trip is $d_{\text {in }}+d_{\text {out }}=10 \mathrm{~km}$.

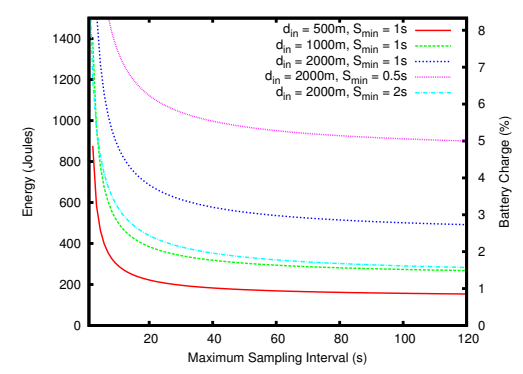

Fig. 6. Total energy of app from analysis.

We note that as the maximum sampling interval is increased, the energy needed by the application reduces rapidly, stabilizing beyond values of $60 \mathrm{~s}$. Further, we note that the energy consumption is sensitive to the congestion zone radius, as this is where a majority of the samples are obtained. For the same reason, the sampling interval within the congestion zone also has a considerable affect on the energy consumed. For our experiments, we used $d_{\text {in }}=2000 \mathrm{~m}$ as we observed that most congestion situations were contained within this stretch. The smart phones used in our experiments had battery energy of about $18000 \mathrm{~J}$ and we set a goal for RTChoke that the application should not use more than $3 \%$ of the battery charge, which amounts to $560 \mathrm{~J}$. Based on this constraint, we choose $S_{\min }=1 s$ and $S_{\max }=120 s$ for our experiments.

\section{B. Hotspot Detection}

Consider a road segment $R$ where a congestion builds up due to an incident, such as an accident, at time $t=0$. We compute the time taken for the server to detect that the segment might be a possible hotspot. Let $S$ be the current sampling interval on $R$ given the location of existing hotspots (derived from Equation 11.

We assume that the server tags the segment as a possible hotspot if it receives $N$ samples. We also assume that the communication of speeds from the application to the server is error-free. We first compute the number of vehicles that are on the road segment as a function of time, and then estimate the time required for the server to receive $N$ samples.

1. Vehicle build-up function: We denote the rate at which the vehicles enter the road segment $R$ by $r_{\text {in }}$ vehicles per unit time. Let $r_{\text {out }}$ be the rate at which the traffic exists the hotspot. We assume that $r_{\text {out }} \leq r_{\text {in }}$ at $R$, and hence the queue dynamics leads to infinite buildup until the traffic regulators defuse the congestion by external means. Our aim

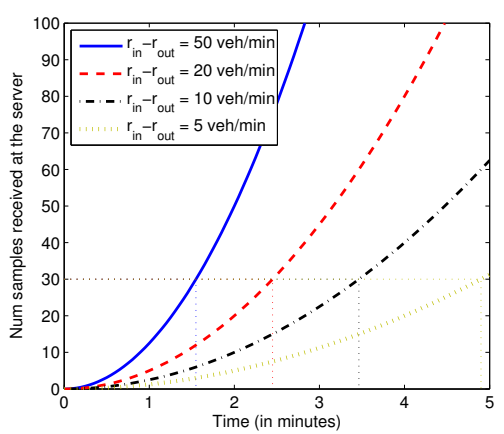

Fig. 7. Time required to detect a hotspot decreases rapidly as $r_{\text {in }}-r_{\text {out }}$ increases

is to estimate the time required to detect congestion buildup, and thus provide a framework to notify flash congestion to travelers and regulators.

The number of vehicles that accumulate on $R$ in time $t$ is given by $c(t)=\left(r_{\text {in }}-r_{\text {out }}\right) t$; the congestion buildup increases linearly.

2. Number of samples at time $t$ : We now estimate the number of samples that the server receives at a given time $t$. Since one car sends the sample every $S$ time units, the expected number of samples at time $t$ from $c(t)$ is $\frac{c(t)}{S}$. Hence, over a period $[0, t]$, the total number of samples received at the server is defined by

$$
n(t)=\int_{0}^{t} \frac{c(x)}{S} d x=\frac{\left(r_{\mathrm{in}}-r_{\mathrm{out}}\right) t^{2}}{2 S} .
$$

The time required for the server to collect $N$ samples to detect if $R$ is a possible hotspot can be determined by equating $n(t)=N$. Hence,

$$
T=\sqrt{\frac{2 N S}{r_{\mathrm{in}}-r_{\mathrm{out}}}} .
$$

Hence, the time required to detect a road segment as a hotspot at the server decreases drastically, as a square root function of differential of the rates at the segment.

Figure 7 shows the time required to detect a hotspot where $N=30, S=S_{\max }=2$ minutes, and for different $r_{\text {in }}$ and $r_{\text {out }}$. Rates are measured in vehicles per minute, and are derived from existing traffic literature [21]. As the difference in the entry and exit rate increases, the detection time for congestion decreases rapidly. Once the server detects the congestion hotspot, regular adaptive sampling can be resumed.

\section{EXPERIMENTAL EVALUATION}

We evaluate RTChoke using two sets of data. The first set was obtained from a pilot study of 12 smart-phone users commuting to and from their work place at TechPark, located in a major city in a developing country, over a period of almost 2 months. The second set was traces of taxicab location information in San Francisco city [8]. 


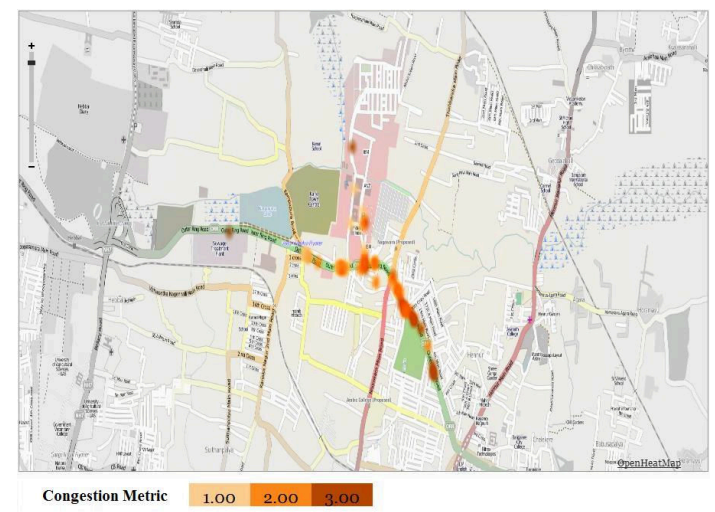

Fig. 8. Congestion detection at TechPark.

\section{A. Congestion at TechPark}

In Section II], Figures 1 and 3, showed that the roads close to TechPark experienced much lower average speeds during peak hours than roads that were farther away and that the observed speeds varied significantly with time of day. In our evaluation, we show: (1) RTChoke can accurately detect congestion hotspots that exhibit low average speeds as well as temporal variation, (2) Effectiveness of adaptive-sampling compared to continuous sampling, and (3) Comparison of the energy footprint of our application with that of Google Maps with traffic updates enabled. For these experiments, we set $d_{i n}=2000 \mathrm{~m}, S_{\min }=1 \mathrm{~s}$, and $S_{\max }=2 \mathrm{~min}$, adhering to the analysis presented in Section IV

1) Real-time Congestion Detection: The TechPark region (highlighted with a blue rectangle in Figure 11 in Section II] hosts multiple organizations employing more than 100,000 people. As noticeable in the figure, the average speeds just outside TechPark are quite low during peak hours due to severe congestion. Based on real-time feeds obtained from smartphone users, we were able to identify segments of congestion and classify them into three levels (level 1 represents low congestion and level 3 represents high congestion). This is shown as a heat-map in Figure 8, demonstrating RTChoke's effectiveness in detecting hotspots.

Another key observation, is that Figure 8 shows that the region inside the TechPark is not congested, although Figure 1 shows the average speeds on these roads to be low (inside the blue rectangle). In fact, these roads are indeed not congested, and the low speeds are due to narrower roads with speed bumps every 20 meters. As speeds are uniformly low and there is no significant difference between the current observed speeds and the maximum reference speed for the road-segments, we do not classify them as congestion hotspots. This is in line with our aim of monitoring only those regions with high variance in speeds, and not waste critical battery energy monitoring areas with uniform speeds (we can afford to obtain samples at a lower frequency). RTChoke was able to distinguish such areas with uniformly low speeds from actual congestion hotspots that exhibit a large deviation between the current observed speeds and the maximum speed for that roadsegment.

In addition to detecting hotspots, high sampling rate near the hotspots ensures that we obtain sufficient samples around the hotspot region to be able to accurately characterize temporal variance at the hotspot as shown in Figure 3

2) Adaptive vs. Continuous Sampling: Next, we evaluate the overhead and accuracy of RTChoke with continuous and adaptive GPS sampling. For the continuous mode, GPS was sampled every second (same as $S_{\min }$ for adaptive sampling). We consider a trip of $15 \mathrm{~km}$ from TechPark to user home. Congestion zone is a circle with $2 \mathrm{~km}$ radius outside the TechPark (as shown in Figure 8).

Figure 9 shows how the sampling interval increases sharply (based on adaptive sampling) as a typical user moved away from the hotspot zone. The sampling period is multiplicatively increased from $S_{\min }$ as the user moves away from the hotspot. As shown in Figure 10, adaptive sampling yields about $9 \times$ improvement in terms of the overhead, captured as the number of GPS samples collected and uploaded.

We now discuss the accuracy of adaptive sampling. We define the accuracy as the time when the user is detected to have entered the congestion zone when using adaptive sampling as compared to the app using continuous GPS sampling. We compare the time when the congestion zone was entered for 3 trips from user home to TechPark (reverse of the above trajectory from office to home) since it is a harder case to detect entrance We observed that the adaptive sampling took additional 45 seconds on an average to detect that the user has entered the congestion zone when compared to the continuous sampling, which is merely $4.8 \%$ of the total time spent by the user in the congestion zone.

3) Energy Footprint: We compare the energy footprint of RTChoke with Google Maps [7] app with real-time traffic updates enabled. To estimate application-specific energy consumption, we use PowerTutor [22], which provides perapplication energy consumption by different components, such as CPU, 3G, and LCD screen. Google maps with traffic updates enabled, can be used to view the status of congestion on different roads. Unlike the adaptive sampling of RTChoke, Google maps samples GPS continuously and uploads location data in real-time to the server. It retrieves traffic information in terms of low-, medium-, and high-speeds from their server(s). The only additional operation performed by Google maps is retrieving map-specific data. RTChoke does not require map-specific information for its functioning. For fairness of comparison, we perform the experiment by running RTChoke and Google maps on two identical HTC Desire smartphones, both carried by the same user. Also, we subtract the LCD screen specific energy consumption from the total energy to measure only the energy consumed by the application.

Figure 11 compares energy consumed by RTChoke and Google maps over time, when a user (carrying both the phones) is moving away from a congestion hotspot. Note that for the first 170 seconds, the user is within the congestion zone, where RTChoke samples and uploads at the peak rate. Thus, RTChoke consumes almost as much energy as Google maps. However, Google map's energy consumption increases almost linearly even beyond the congestion region, but energy for 


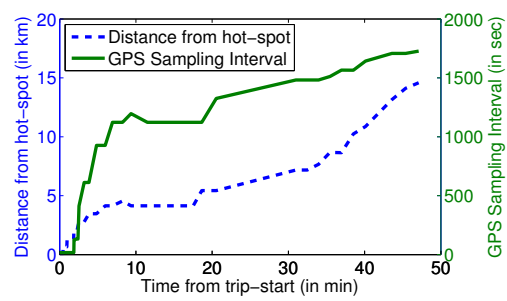

Fig. 9. Adaptation of GPS sampling interval as user is moving away from a hotspot.

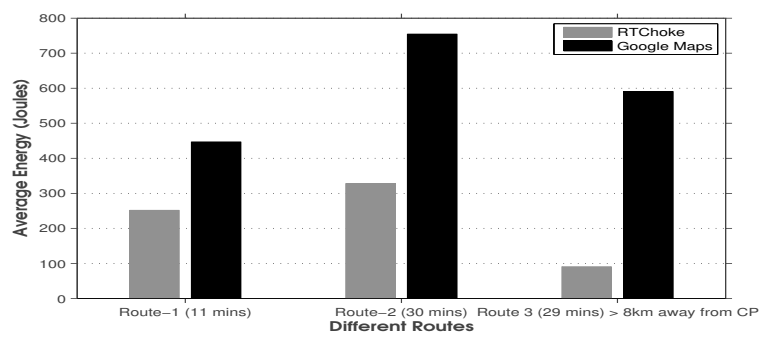

Fig. 12. Fnerov consumntion on RTChoke and Gonole Maps.

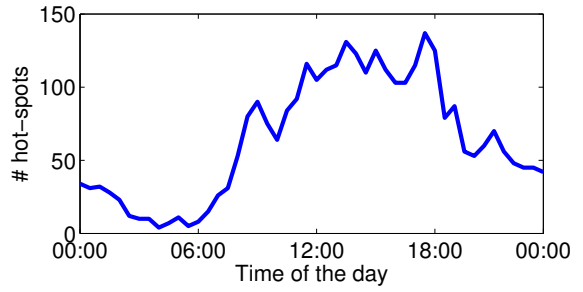

Fig. 13. SFO: Hotspots vary with time-of-day

RTChoke grows much slower because of adaptive sampling.

We also measured the total uplink traffic from RTChoke and Google maps (graph omitted in the interest of space). The total bytes uploaded by Google maps was $85.5 \mathrm{~KB}$, whereas RTChoke uploaded $19.36 \mathrm{~KB}$ data, a reduction in network usage by about 4.5 times.

To avoid route-specific biases, we also compared the average energy consumption for three different routes with different travel durations as shown in Figure 12. It can be observed that the difference in energy consumption between Google maps and RTChoke is higher when the duration of travel away from a congestion hotspot is higher, as demonstrated by Route- 1 and Route- 2 . When the user is traveling a longer distance outside the congestion hotspot ( $8 \mathrm{~km}$ away for Route3 ), adaptive sampling yields an order of magnitude lower energy footprint compared to that of Google maps.

\section{B. Congestion in San Francisco}

We analyzed location information from 200 cabs across 3300 road segments in San Francisco city using the CRAWDAD data [8]. Each cab reports GPS coordinates and timestamp once every minute. We process this data as explained in Section III to estimate the congestion in SFO city.

We use the temporal speed data per road-segment to compute the congestion levels in SFO city. The median average speed on road-segments is around $45 \mathrm{kmph}$ (or $28 \mathrm{mph}$ ). Fig. 13 shows the temporal variance of hotspots in SFO city;

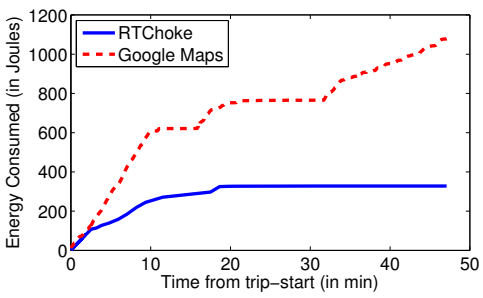

Fig. 11. Comparison of total energy consumed by RTChoke and Google maps.

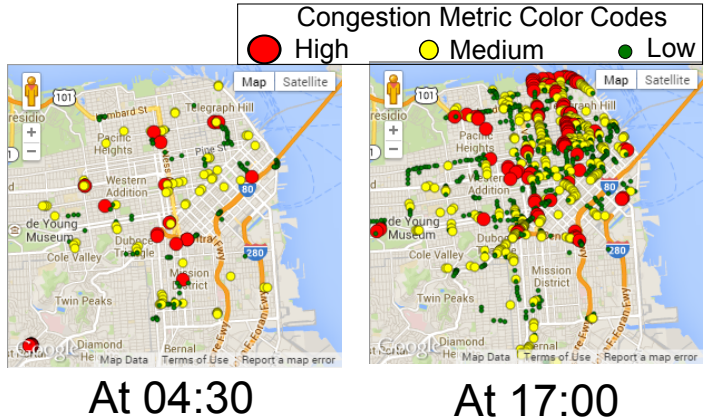

Fig. 14. Off-peak and Peak hour congestion heat-map

the number of hotspots vary with the time-of-day showing peak and off-peak traffic. Fig. 14 shows the congestion heatmap at peak traffic (at 17:00), with hotspots marked in red. Only 137 roads (approximately $4 \%$ of the all roads sampled) were tagged as hotspots, where users should expect large delays due to lower speeds. During the off-peak hours (at 04:30) the number of congested road-segments was even smaller. Fig. 15 shows the spatio-temporal variations of SFO congestion hot-spots at different times of the day.

Similar to TechPark data, we observed that hotspots demonstrated a much larger variation in speeds when compared to the other road-segments. In order to measure how the road-speeds vary in congestion hotspots, we compute the Normalized Deviation (ND) of each road-segment, defined as the ratio of the standard deviation to the average speed on the roadsegment. Fig. 16 plots the CDF of ND for hotspots and non hotspots. The figure shows that hotspots have larger values of $\mathrm{ND}$, and hence larger temporal variation.

Fig. 17 shows the trajectories followed by two cabs at 17:05 and $17: 16$ on the same day. It also shows the average speed of the roads between 17:00 to 17:30. A square marks the congestion hotspot. Note that the cabs were traveling much faster outside the hotspot than closer to it.

We recommend actively monitoring a small number of hotspots instead of all road segments based on our observation that deviation in speeds is higher at hotspots and lower at nonhotspots. For the non hotspots, average speeds at the given time-bin over all history can be used as an approximate speed. Such selective monitoring of hotspots with high frequency can be used for low-complexity and energy-aware congestion monitoring for applications (e.g., alerting users about possible congestion and suggesting alternate routes). Adaptive Sam- 


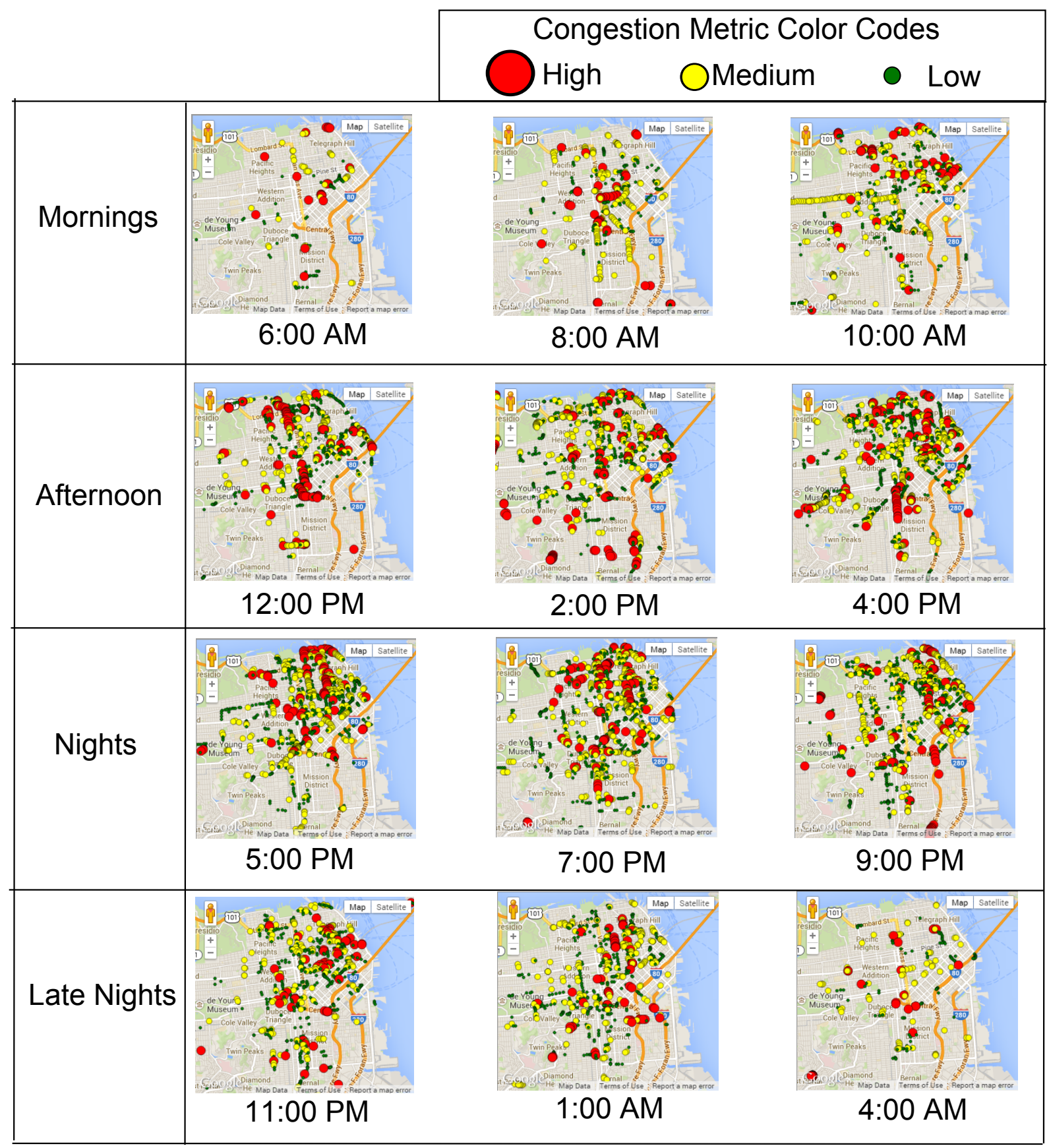

Fig. 15. Spatio-temporal variation of congestion hot-spots in SFO

pling approach suggested in Section III-C2 utilizes this insight to monitor frequently at the hotspots, and to save energy by low-rate monitoring at other road-segments.

Flash congestion detection: We now compare the time required to detect flash-congestion. We simulate flash-congestion caused by an accident, in which all the cars approaching the accident location get blocked. We use the real location traces for cars from the San Francisco cabs data set. We assume that multiple cars (as varied on $\mathrm{X}$-axis) need to report low speeds to detect congestion reliably. We consider $S_{\min }=1$ minute (the minimum granularity of samples in the data set) and $S_{\max }=2$ minutes. Since there was no congestion before, we use $S_{\max }$ as the cars' sampling interval. We consider two types of alerting modes: Regular and Expedited. In the Regular mode, cars do not change their sampling interval until congestion is detected reliably. On the other hand, in the Expedited mode, the server declares that a reported location is a possible congestion hotspot, if even a single car notifies lower speed than threshold. In such a case, the server notifies the other cars approaching the same possible congestion point to sample at peak rate (at $S_{\min }$ ).

Fig. 18 plots the time required to reliably detect flash- 


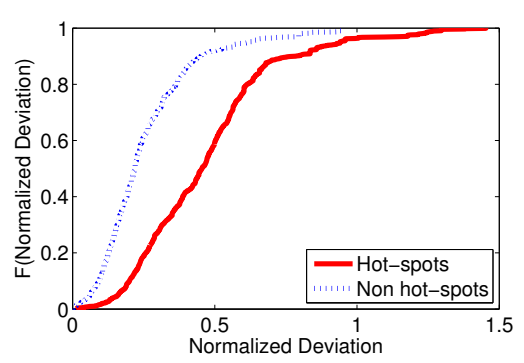

Fig. 16. Deviation observed on hotspots are larger than those observed on non hotspots

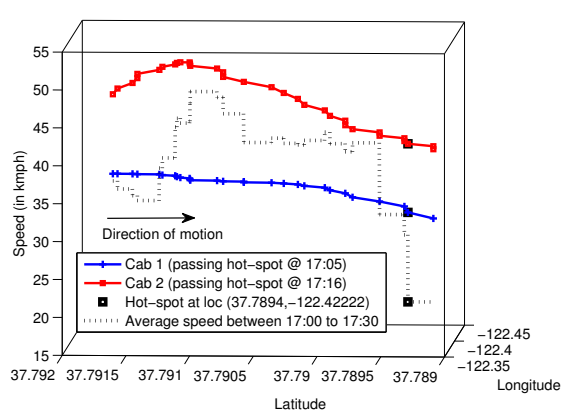

Fig. 17. Two trajectories intersecting at a hotspots

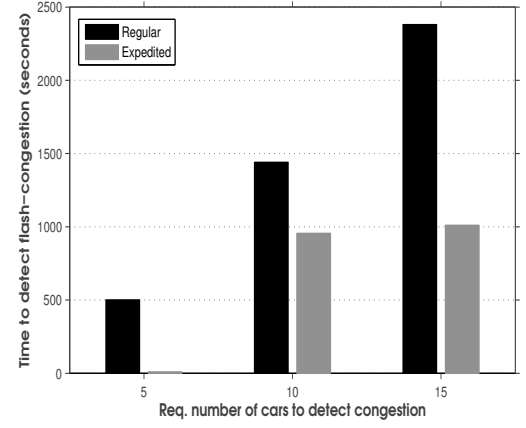

Fig. 18. Time to detect flash congestion. congestion after the first car has notified a lower-speed value than the congestion threshold. It can be seen that the time to detect flash congestion can be reduced by up to $50 \times$ using Expedited alerting mode. The significant increase in congestion detection time from that for 5 versus 10 or 15 cars is due to the cabs arrival rate in the dataset.

\section{CONCLUSION}

In this paper, we show that in a traffic monitoring system built over mobile sensors, automatically detecting and monitoring congestion hotspots can significantly reduce the overall overhead of the system, without compromising on the accuracy, in comparison, to a system with fine-grained periodic monitoring. The solution, RTChoke, requires minimal user intervention, and optimizes both battery power and network bytes used for data uploads. We believe that detecting hotspots and monitoring congestion just based on hotspots makes participatory sensing traffic applications efficient by coupling collection and use of data.

\section{REFERENCES}

[1] Press Information Bureau, Govt. of India, "http://pib.nic.in/archieve/factsheet/fs2000/roads.html."

[2] Urban Mobility Report 2011, Texas Transportation Institute, "http://mobility.tamu.edu/ums/report/."

[3] Mobile Millennium Project, "http://traffic.berkeley.edu/."

[4] A. Thiagarajan, J. Biagioni, T. Gerlich, and J. Eriksson, "Cooperative transit tracking using smartphones," in ACM Sensys, 2010, pp. 85-98.

[5] A. Thiagarajan, L. Ravindranath, K. LaCurts, S. Madden, H. Balakrishnan, S. Toledo, and J. Eriksson, "Vtrack: Accurate, energy-aware road traffic delay estimation using mobile phones," in ACM Sensys, 2009.

[6] P. Mohan, V. Padmanabhan, and R. Ramjee, "Nericell: Rich monitoring of road and traffic conditions using mobile smartphones," in $A C M$ Sensys, 2008, pp. 323-336.

[7] Google Maps, "https://maps.google.com/."

[8] M. Piorkowski, N. Sarafijanovoc-Djukic, and M. Grossglauser, "A Parsimonious Model of Mobile Partitioned Networks with Clustering," in COMSNETS, January 2009.

[9] B. Kerner, "Three-phase traffic theory and highway capacity," Physica A: Statistical Mechanics and its Applications, vol. 333, 2004.

[10] B. Kerner, H. Rehborn, M. Aleksic, and A. Haug, "Recognition and tracking of spatial-temporal congested traffic patterns on freeways," Elsevier Transportation Research, vol. 12, no. 5, 2004.

[11] INRIX - Go Anywhere, "http://www.inrix.com/."

[12] J. Yoon, B. Noble, and M. Liu, "Surface street traffic estimation," in ACM Mobisys, 2007, pp. 220-232.

[13] R. Ganti, N. Pham, H. Ahmadi, S. Nangia, and T. Abdelzaher, "Greengps: A participatory sensing fuel-efficient maps application," in ACM Mobisys, 2010, pp. 151-164.
[14] J. Eriksson, L. Girod, B. Hull, R. Newton, S. Madden, and H. Balakrishnan, "The pothole patrol: Using a mobile sensor network for road surface monitoring," in ACM MobiSys, 2008.

[15] R. Balan, K. Nguyen, and L. Jiang, "Real-time trip information service for a large taxi fleet," in ACM Mobisys, 2011, pp. 99-112.

[16] J. Burke, D. Estrin, M. Hansen, A. Parker, N. Ramanathan, S. Reddy, and M. Srivastava, "Participatory sensing," in Workshop on World-SensorWeb, 2006, pp. 117-134.

[17] Waze, "http://www.waze.com/."

[18] Open Street Map, "http://www.openstreetmap.org/."

[19] P. Newson and J. Krumm, "Hidden markov map matching through noise and sparseness," in ACM SIGSPATIAL GIS, 2009.

[20] M. Mun, S. Reddy, K. Shilton, N. Yau, J. Burke, D. Estrin, M. Hansen, E. Howard, R. West, and P. Boda, "Peir, the personal environmental impact report, as a platform for participatory sensing systems research," in ACM Mobisys, 2009, pp. 55-68.

[21] V. Jain, A. Sharma, and L. Subramanian, "Road traffic congestion in the developing world," in ACM DEV, 2012, p. 11.

[22] PowerTutor, "http://powertutor.org/." 